\title{
Electrochemical Sensors for Environmental Monitoring in Cities
}

\author{
R. L.Jones ${ }^{a^{*}}$, G.Stewart ${ }^{a}$, O.Popoola ${ }^{a}, I_{\text {Mead }}{ }^{a}$ and J.Saffell ${ }^{b}$ \\ ${ }^{a}$ Centre for Atmospheric Science, Department of Chemistry, \\ University of Cambridge, Lensfield Road, Cambridge, CB2 1EW, U.K. \\ ${ }^{b}$ Alphasense, Sensor Technology House, 300 Avenue West, Skyline 120, \\ Great Notley, CM77 7AA, U.K.
}

\begin{abstract}
We demonstrate that miniature, low-cost electrochemical gas sensors, traditionally used for sensing at parts-per-million (ppm) mixing ratios can, when suitably configured and operated, be used for partsper-billion $(\mathrm{ppb})$ level studies for gases relevant to urban air quality. The results show the sensors to be high precision and highly linear in the ppb rangeand in general highly selective, although there are some significant cross-interferences. Comparisons of the sensors with co-located reference instruments show good agreement on both short and long timescales, demonstrating their utility for urban air quality monitoring.

We have also integrated multiple sensors with GPS (location) and GPRS (real time data transmission) to create multi-sensor nodes fordeployment as components of sensor network systems. These have been deployed in substantial numbers as both mobile and static networks systems, providing unprecedented real time spatial and statistical information on urban air quality.

We have demonstrated the utility of electrochemical sensors to provide air quality measurements traditionally viewed as only achievable by costly and sparse fixed site monitoring stations. We have shown that this technology can also achieve measurement densities, ready scalability, portability and low cost unachievable by current fixed site networks which collectively promises to add hugely to the capability for monitoring urban air quality for legislation and personal exposure.
\end{abstract}

Keywords: Electrochemical gas sensors; sensor networks; urban air quality; environmental measurements 